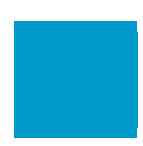

\title{
Führungspersonen haben heute viel mehr Verantwortung und Gestaltungsmöglichkeiten
}

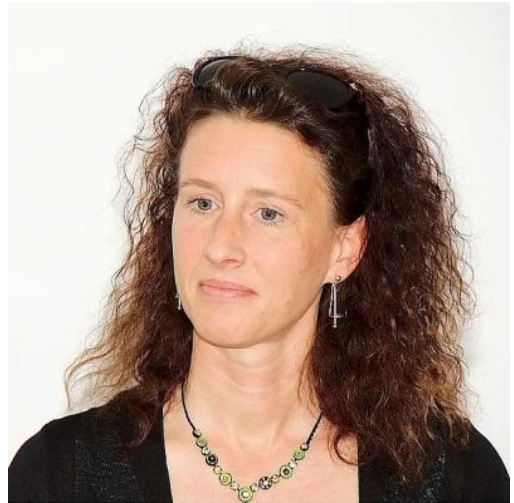

Foto: privat
Petra Heißenberger leitet das Zentrum Leadership an der Pädagogischen Hochschule Niederösterreich (PH NÖ) und ist unter anderem für Fort- und Weiterbildungen von Schulleitenden und an schulischer Führung interessierten Personen zuständig. Seit 2013 hat sie die Hochschulprofessur für Schulmanagement inne. Sie ist in verschiedenen Arbeitsgruppen und mit der Implementierung von Projekten befasst. In der Forschung liegen ihre Schwerpunkte in folgenden Themenfeldern: Schulautonomie (Projekt INNOVITAS), Führungspersönlichkeiten und deren Motivation für Weiterbildungen und ihre Führungstätigkeit.

An der Pädagogischen Hochschule Niederösterreich (PH NÖ) gibt es in der Weiterbildung mehrere Hochschullehrgänge (HLG) im Bereich Schulmanagement und vielfältige Angebote im Bereich der Fortbildung schulischer Führungskräfte. Was sich derzeit ändert, ist Thema des Interviews, welches für den Schulführungsblog der Pädagogischen Hochschule Zürich (PHZH) geführt wurde. Die PHZH gestaltet und flexibilisiert momentan ihre Schulleitungsausbildung neu, die im Sommer 2022 starten soll. Ein wesentliches Element ist ein flexibler Einstieg in die Führung als Teacher Leader.

\section{In Österreich wird zur Zeit die Schulleitungsausbildung auf neue Beine gestellt. Welche Hochschullehrgänge (HLG) müssen besucht werden?}

Petra Heißenberger: Momentan gilt in Österreich noch die Regelung, dass eine Lehrperson zuerst als Führungskraft ernannt und auf Ersuchen des Dienstgebers von der PH NÖ zum HLG "Schulmanagement" eingeladen wird. Dieser HLG hat ein Ausmass von 12 ECTS-Punkten und dauert vier Semester. Diese Regelung gilt seit 1996 und wird noch bis zum 1. Januar 2023 gelten. Danach kommt die neue Ausbildung zum Zuge.

Bereits heute können Lehrer*innen den HLG „Schulen professionell führen Vorqualifikation" besuchen. Dieser HLG hat ein Ausmass von 20 ECTS-Punkten und ist ab 
2023 die Voraussetzung, um sich auf eine ausgeschriebene Führungsfunktion in einer Schule zu bewerben. Er dauert zwei Semester und wird berufsbegleitend organisiert. Nach der Übernahme einer Führungsfunktion müssen ergänzend zur Vorqualifikation noch weitere 40 ECTS-Punkte des HLGs „Schulen professionell führen“ absolviert werden. Dieser dauert vier Semester.

Das ist doch eine deutliche Ausweitung der Ausbildung zur Schulleiterin oder zum Schulleiter.

Ja, das ist es tatsächlich. Einerseits hat sich der Umfang der Ausbildung verfünffacht. Anstelle von 12 ECTS-Punkten und vier Semestern dauert die Ausbildung nun insgesamt sechs Semester und hat einen Umfang von 60 ECTS-Punkten.

Neu ist auch, dass die Ausbildung zweigeteilt ist. Einerseits gibt es eine Vorqualifikation für alle Interessierten. Am Ende des HLGs "Schulen professionell führen - Vorqualifikation“ sollen die Absolvent*innen Klarheit darüber haben, ob sie sich für eine schulische Führungsfunktion bewerben wollen oder nicht. Sobald jemand tatsächlich in der Führung tätig ist, ist der zweite Teil der Qualifikation zu absolvieren.

\section{Vor dem Job und „on the job”}

\section{Welche Gründe haben zu dieser Veränderung geführt?}

Einerseits hatten wir Rückmeldungen von schulischen Führungskräften, dass sie sich eine Ausbildung bereits vor der Bewerbung wünschen würden. So lernen sie die Aufgabe kennen und können sich auf diese vorbereiten. Andererseits hatten wir die Rückmeldung, dass eine Begleitung „on the job" sehr wesentlich ist und eine Vorqualifikation nicht ersetzen kann.

Gleichzeitig hat sich das Aufgabenprofil der Schulleitung in den letzten zehn Jahren sehr verändert. Führungspersonen haben viel mehr Verantwortung und Gestaltungsmöglichkeiten. Um diese Aufgaben kompetent erfüllen zu können, benötigen sie eine fundierte Ausbildung und Begleitung.

\section{Welche Inhalte sind in der Vorqualifikation zentral?}

Den besten Eindruck erhält man, wenn man sich das Curriculum anschaut. Dieses ist auf der Homepage der PH NÖ einsehbar. Es geht unter anderem um das eigene Führungsverständnis, um die Themenfelder Organisation, Personal und Qualität. 


\section{Anspruch und Vielfalt}

Und welche Inhalte sind in der in der zweiten Phase bei der Übernahme einer Schulleitungsfunktion zentral?

Bei diesem HLG ist das Detailcurriculum gerade in Entwicklung, sodass dieses momentan noch nicht auf der Homepage abgerufen werden kann. Einige Themenfelder wurden bereits definiert: Professionsmodelle, Professionsverständnis, evidenzbasierte Steuerung, aktuelle bildungspolitische Herausforderungen und Chancen, Selbst- und Zeitmanagement, Schule im Feld, Führungstheorien, Organisationsentwicklung, Personalentwicklung, Qualitätsmanagement, Schul- und Dienstrecht, Projektmanagement, datenbasierte Unterrichtsentwicklung, datenbasierte Schulentwicklung, die vernetzte Schule, Kommunikation und Konfliktmanagement. Wie die Länge der Liste zeigt, ist die Leitung einer Schule anspruchsvoll und vielfältig, so muss es auch die Qualifizierung sein.

\section{An der PH NÖ kann man im Bereich Schulmanagement auch einen Masterabschluss erzielen. Was unterscheidet den Master zu den anderen Schulleitungsausbildungen?}

Der HLG mit Masterabschluss „Schulmanagement: Professionell führen - nachhaltig entwickeln" dauert sechs Semester und hat unter anderem qualitative und quantitative Forschungsmethoden zum Inhalt. Das Curriculum dazu ist ebenfalls auf der Homepage der PH NÖ einsehbar.

Welche Führungspersonen besuchen diesen HLG? Und warum absolvieren sie ein solch anspruchsvolles Studium?

Wir stehen mit unseren Absolvent*innen im Kontakt. Sie berichten über ihre vielfältige Motivation, den HLG zu besuchen: Einerseits ist es das Interesse, Anforderungen an Schulleitungen professionell zu reflektieren mit dem Ziel, die Leitung einer Schule zu übernehmen oder autonomer zu gestalten. Die persönliche Wissenserweiterung und Weiterentwicklung im Kontext Leadership stellt eine weitere wichtige Motivation dar.

Ausserdem bietet der HLG die Möglichkeit, sich theoriefundiert im Bereich des qualitativen und quantitativen Forschens zu vertiefen und den eigenen Schulstandort datenbasiert weiterentwickeln zu können. Letztlich sollen die Inhalte ja bei den Schüler*innen ankommen.

Mit Petra Heißenberger (PH NÖ) sprach Niels Anderegg (PHZH) 